\title{
Creating a Virtual Support Group in an Interactive Narrative: A Companionship Game for Cancer Patients
}

Alice Bowman

This is the Author Accepted Manuscript of a conference paper published in Interactive Storytelling, 11th International Conference on Interactive Digital Storytelling, ICIDS 2018, Dublin, Ireland, December 5-8, 2018, Proceedings.

The final authenticated version is available online at: https://doi.org/10.1007/978-3-030-04028-4 80 


\title{
Creating a Virtual Support Group in an Interactive Narrative: A Companionship Game for Cancer Patients
}

\author{
Alice Bowman* \\ Abertay University \\ Bell Street, Dundee \\ DD1 1HG
}

\begin{abstract}
Over one in five cancer patients are affected by feelings of loneliness [1]. This paper proposes a text-based game aimed at affected cancer patients, in which the player develops relationships with non-player characters in the context of a fictitious cancer support group. This would be designed with the aim of alleviating patient loneliness and fostering a sense of companionship. This work is part of an interdisciplinary project led by Abertay University and partnered with Macmillan Cancer Support, exploring the ways in which interactive storytelling can be used to support cancer patients. The game's narrative and characters will be designed drawing on real patient experiences gathered in interviews. The game will go through several iterations of feedback and rewriting in response to focus groups held with patients and healthcare professionals.
\end{abstract}

\section{Introduction}

Cancer patients are often affected by feelings of loneliness and isolation. Macmillan Cancer Support published findings in 2014 that estimated more than one in five cancer patients (22\%) experience loneliness [1]. These findings indicate that patients who have reported loneliness are three times more likely to struggle to follow their treatment plan than those who are not lonely. The research work described in this paper is part of an interdisciplinary project at Abertay University in partnership with Macmillan Cancer Support, the goal of which is to support cancer patients through interactive storytelling. The project detailed here aims to fulfil this goal through the development of a text-based game aimed at cancer patients affected by loneliness. The game's narrative will focus on developing positive relationships between the player and non-player characters in the context of a fictitious cancer support group, in order to establish a sense of companionship.

*I would like to thank Dr Dayna Galloway, Prof James Bown and Dr Sonia Fizek for their guidance and feedback in writing this paper. 


\section{Research Questions}

The overall project asks how interactive storytelling can be employed to support cancer patients. There are a multitude of problem areas in the lives of cancer patients that could be supported by digital tools, the problem areas of loneliness and isolation were chosen as the focus for this game for two reasons. Firstly, because of how widely reported these feelings are among cancer patients and the severity with which they can impact quality of life and prognosis [1]. Secondly, based on existing games and the relationships players are able to develop with characters [2], there is a strong case for hypothesising that a character focused text-game will be able to reduce loneliness and create feelings of support, and that such a game will be deliverable within the scope of this project.

The thesis being written parallel to the development of this game will not just focus on games for healthcare or games designed to combat loneliness. It will be centred around the question of how to create compelling, emotionally engaging game characters in a serious narrative context - with the proposed game being used as a case study. Within the bounds of this research a serious game narrative will refer to both serious games and other types of game in which the narrative features sensitive and/or mature themes. In Serious Games: Games That Educate, Train and Inform [3] serious games are defined as 'a game in which education (in its various forms) is the primary goal rather than entertainment.' The need for engaging and meaningful characters in this type of game arises from a necessity of the player being engaged and invested in the play experience in order for the game to achieve its goals.

\section{Methodology}

The first step in planning this project was assessing the user base in order to determine how a game would be able to provide support, and what issues to focus on. This was done through evaluation and thematic analysis of posts on cancer-related public Internet forums, patient blogs and video blogs, resources and information directed at patients, and various media aimed at cancer patients or telling the stories of cancer patients.

Based on this research user personas [4] were created. Patient statistics and the gathered materials on patient experiences were used together to design a sample of fictional cancer patients to represent the user base. Early design concepts for potential games were created in response to these personas, and ultimately a game to support lonely and isolated patients was chosen for development due to this preliminary research highlighting the prevalence and severity of these problems.

Semi-Structured interviews will be carried out with cancer patients, discussing their experiences since diagnosis. These interviews will be facilitated by Macmillan Cancer Support, and the information gathered here will be used to inform the narrative of the game. This will be done in order to gain a deeper understanding of patient lifestyle, and to give a clearer voice to the patients being represented in the game. In order for the game's 
fiction to be realistic and engaging patients must feel that the narrative is an accurate representation of their experiences.

With a design shaped by the data gathered in these interviews, the initial prototype of the game will be created using Twine $2[5]$. After the prototype is developed and playtested internally it will be presented to a focus group comprised of stakeholders with a background in healthcare. The purpose of this focus group will be to assess whether the game is at a stage to be used by patients, and to discuss any concerns the stakeholders raise. Patient wellbeing will remain the priority throughout the project, and so any concerns or criticism at this stage will be addressed in a new iteration of the game.

When the prototype is at a stage deemed suitable for patient playtesting another focus group will be created consisting of cancer patients. This group will be asked to complete a questionnaire assessing emotional state before and after playing the game, and will also be interviewed in order to gather feedback on the game. Patients will be asked to report back on playing the game over a period of two weeks, as well as giving feedback following a single play session. This data will be used as a preliminary indicator of the game's effectiveness, with patient feedback being incorporated into changes in the next iteration of the game.

Subject to positive indicators of the game's effectiveness the game will be launched on a wider scale. The game will be made available on iOS, android and the Internet. Data from these platforms will not be analysed beyond reference to player statistics due to the scale of the data. Parallel to this digital launch, the game will also be launched locally, with installations at community access points. Further evaluation of the game's effectiveness will be carried out in situ at these installations.

\section{Prototype Design Outline}

The thematic analysis carried out on Internet forum posts indicated that patients felt a deeper sense of support from others who had been through the same experiences and treatment, some writing that they felt only others who had been through these experiences could understand. The decision to set the game in the context of a support group was based off of this, the cast of non-player characters will be going through similar experiences to the player, this is intended to create a sense of empathy and camaraderie.

The dialogue options will be pre-defined rather than freeform text due to the scope of the project and limitations on resources. These dialogue options will branch the narrative, changing the response the player receives dependant on choice, the structure will then reconverge. Tracked variables will define different aspects of relationships between the player character and non-player characters, tracking their familiarity and dynamic, as well

as accounting for the demonstrated personality of the player character. Passages of the game will vary depending of the state of certain variables.

The game will play in real time with game events following a pre-defined schedule, the player will receive notifications when a game event is playable or a non-player character 
tries to contact them. This aspect of the design draws inspiration from the Lifeline [6] series and Mystic Messenger [7]. Pacing the game in real time permits the game to be designed to fit with play patterns expected from the user persona, and will add a sense of realism to the game and interactions with non-player characters.

\section{$5 \quad$ Next Steps}

At the time of writing, patient interviews are scheduled to take place in September 2018. Once this data has been analysed writing for the prototype will begin. A working build of the prototype will be presented at the ICIDS 2018 Doctoral Consortium.

The version of the prototype will be a work in progress and writing for the initial iteration is scheduled to continue until February 2019. At this stage, feedback on the writing, branching structure and overall narrative of the design would be highly appreciated. The continued timescale of work on the prototype means that feedback and critique can be incorporated into later iterations.

Further steps on the project will include the cycle of playtesting, gathering feedback and iterating on the prototype, as detailed in the above methodology section. By working closely with cancer patients and healthcare professionals to hone the design, the project aims to deliver an interactive narrative that can quantifiably alleviate loneliness in cancer patients.

\section{References}

[1] Macmillan (2014) Lonely cancer patients three times more likely to struggle with treatment. Available at: https://www.macmillan.org.uk/aboutus/news/latest_ news/lonelycancerpatientsthreetimesmorelikelytostrugglewithtreatment. $\operatorname{aspx}$ (Accessed: 18 July 2018)

[2] Waern, A. (2010) “'I'm in love with someone that doesn't exist!!” Bleed in the Context of a Computer Game.' Paper from the Nordic DiGRA Conference, Stockholm. Available at: http://www.nordic-digra.org/nordicdigra2010_submission_18.pdf (Accessed: 18 July 2018)

[3] Chen, S. and Michael, D. (2005) Serious Games: Games That Educate, Train and Inform. Boston: Course Technology PTR.

[4] Adlin, T. and Pruitt, J. (2005) The Persona Lifecycle: Keeping People in Mind Throughout Product Design. Massachusetts: Morgan Kaufmann.

[5] Chris Klimas (2015) Twine 2 (Version 2.2.1) [Computer program] Available at: https://twinery.org/2/\#!/stories (Accessed: 20 July 2018) 
[6] 3 Minute Games (2015) Lifeline - iOS [Mobile game]. Big Fish Games.

[7] Cheritz (2016) Mystic Messenger - iOS [Mobile game]. Cheritz Co., Ltd 